\title{
Mass Stripping in Dwarf Spheroidal Galaxies and $\omega$ Cen
}

\author{
Takuji Tsujimoto \\ National Astronomical Observatory, Mitaka-shi, Tokyo 181-8588, Japan \\ Toshikazu Shigeyama \\ Research Center for the Early Universe, Graduate School of Science, \\ University of Tokyo, Bunkyo-ku, Tokyo 113-0033, Japan
}

\begin{abstract}
The stellar abundance pattern of neutron-capture elements such as $\mathrm{Ba}$ is used as a powerful tool to infer how star formation proceeded in dwarf spheroidal (dSph) galaxies. It is found that the abundance correlation of $\mathrm{Ba}$ with $\mathrm{Fe}$ in stars belonging to $\mathrm{dSph}$ galaxies have a feature similar to the Ba-Fe correlation in Galactic metal-poor stars. The common feature of these two correlations implies that dSph stars formed from gas with a velocity dispersion of $\sim 26 \mathrm{~km} \mathrm{~s}^{-1}$. This velocity dispersion together with the stellar luminosities strongly suggests that dark matter dominated dSph galaxies. The tidal force of the Milky Way links this velocity dispersion with the currently observed value $\leq 10 \mathrm{~km} \mathrm{~s}^{-1}$ by stripping the dark matter in $\mathrm{dSph}$ galaxies. We also investigate the ram pressure exerted on the gas in $\omega$ Cen. It is found that the ram pressure is not strong enough to strip the gas but is expected to induce a bulk motion of the gas during the star formation epoch which is compatible with the recent observed finding and then the remaining gas after the star formation is likely to be stripped due to the gradual increase in the gas density in the forming Galactic disk.
\end{abstract}

\section{Stripping the dark matter in $\mathrm{dSph}$ galaxies}

The correlation of $[\mathrm{Ba} / \mathrm{Fe}]$ with $[\mathrm{Fe} / \mathrm{H}]$ for $\mathrm{dSph}$ stars is found to be similar to that for Galactic metal-poor stars if the metallicities $[\mathrm{Fe} / \mathrm{H}]$ of $\mathrm{dSph}$ stars are shifted by the amount $\Delta[\mathrm{Fe} / \mathrm{H}]=-0.6$ dex. The metallicities of metal-poor stars are determined by two factors: how much mass of heavy elements a SN supplies and how much mass of interstellar matter (ISM) was eventually swept by a single SN explosion. The former is determined exclusively by each SN. On the other hand, the latter quantity is influenced by the environment such as the velocity dispersion $\sigma_{\mathrm{v}}$ and density $n$ of the ISM. If SNe in the Milky Way and those in dSph galaxies are similar, different environments should give rise to the $\Delta[\mathrm{Fe} / \mathrm{H}]$ mentioned above. Since the mass $M_{\mathrm{sw}}$ swept up by an SN is much more sensitive to the velocity dispersion than to the density; $M_{\mathrm{sw}} \propto$ $\sigma_{\mathrm{v}}^{-9 / 7} n^{-0.062}$ (Shigeyama \& Tsujimoto 1998), it is likely that a larger velocity dispersion in $\mathrm{dSph}$ galaxies at the star formation epoch enhanced the stellar 
$[\mathrm{Fe} / \mathrm{H}]$. Quantitatively, $\Delta[\mathrm{Fe} / \mathrm{H}]=-0.6$ dex corresponds to a velocity dispersion of $\sigma_{\mathrm{v}} \sim 26 \mathrm{~km} \mathrm{~s}^{-1}$. The velocity dispersion $\sigma_{\star}$ of stars must have a similar value in equilibrium configuration. The value of $\sigma_{\star}$ measured in Galactic dSph galaxies is about $9 \mathrm{~km} \mathrm{~s}^{-1}$. These two significantly different velocity dispersions can be connected in the context of a galaxy losing mass by the tidal force of the Milky Way. A larger velocity dispersion at the star formation epoch inferred from the elemental abundance pattern suggests that the total mass of the dSph galaxy at that time must be $\sim 25$ times larger than at present.

\section{Ram pressure stripping of the gas in $\omega$ Cen}

During the star formation phase over a few Gyrs, the proto-cluster cloud for $\omega$ Cen passed repeatedly through the forming Galactic disk. At that time, the gas in the cloud was accelerated by the ram pressure. This ram pressure stripping was not efficient during the star formation phase in $\omega$ Cen because the gas density in the forming Galactic disk during the first few Gyrs is quite small, which is attributed to a slow formation of the Galactic disk inferred from the chemical evolution in the solar neighborhood (Yoshii, Tsujimoto, \& Nomoto 1996). The gas density $\rho_{\text {disk }}$ of the forming disk at the age of 2 Gyr for instance is estimated to be $\sim 1 / 4$ of the present density. From the present $\rho_{\text {disk }}$ of $\sim 0.04$ $M_{\odot} \mathrm{pc}^{-3}$ and the gas density $\rho_{\omega}$ of the proto-cluser cloud for $\omega$ Cen during the late star formation phase of $\sim 2 M_{\odot} \mathrm{pc}^{-3}$, the timescale $t_{\mathrm{rp}}$ for the ram pressure stripping of $\omega$ Cen, defined as $t_{\mathrm{rp}} \approx R / v\left(2 \rho_{\omega} / \rho_{\text {disk }}\right)^{1 / 2}$, is $\sim 10^{7} \mathrm{yr}$, where $R$ and $v$ are the radius $(\sim 100 \mathrm{pc})$ and the velocity $\left(\sim 200 \mathrm{~km} \mathrm{~s}^{-1}\right)$ of the proto-cluster cloud for $\omega$ Cen. On the other hand, the timescale $t_{\text {pass }}$ for the passage through the forming disk is estimated to be $\sim 3 \times 10^{6} \mathrm{yr}$, assuming the present disk scale height of $350 \mathrm{pc}$. Therefore the ram pressure is not strong enough to strip the gas, that is, the ram pressue could not overcome the gravitational attraction of the cloud. The gradual increase in the gas density in the forming disk has made $t_{\mathrm{rp}}$ comparable to $t_{\mathrm{pass}}$ in the next few Gyrs. As a result, the remaning gas after the final episode of star formation in $\omega$ Cen is likely to be stripped by the interaction with the forming Galactic disk.

The ram pressure further leads to a bulk motion of the gas from which stars were formed. In this framework, it is expected that the bulk motion of metalrich population is larger than that of metal-poor population. The increasing density of the disk exerted a greater ram pressure to the proto-cluster cloud at a later passage leading to a larger bulk motion. It is indeed consistent with the observed finding (Ferraro, Bellazzinni, \& Pancino 2002).

\section{References}

Ferraro, F. R., Bellazzini, M., \& Pancino, E. 2002, ApJ, 573, L95

Shigeyama, T., \& Tsujimoto, T. 1998, ApJ, 507, L135

Yoshii, Y., Tsujimoto, T., \& Nomoto, K. 1996, ApJ, 462, 266 